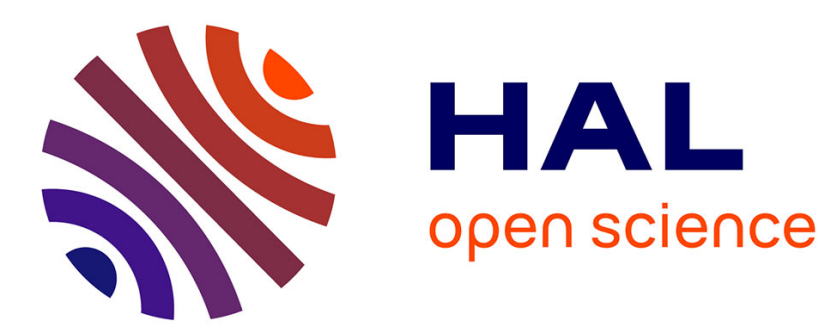

\title{
Rectification of images for binocular and trinocular stereovision
}

\author{
Nicholas Ayache, C. Hansen
}

\section{To cite this version:}

Nicholas Ayache, C. Hansen. Rectification of images for binocular and trinocular stereovision. RR0860, INRIA. 1988. inria-00075694

\section{HAL Id: inria-00075694 https://hal.inria.fr/inria-00075694}

Submitted on 24 May 2006

HAL is a multi-disciplinary open access archive for the deposit and dissemination of scientific research documents, whether they are published or not. The documents may come from teaching and research institutions in France or abroad, or from public or private research centers.
L'archive ouverte pluridisciplinaire HAL, est destinée au dépôt et à la diffusion de documents scientifiques de niveau recherche, publiés ou non, émanant des établissements d'enseignement et de recherche français ou étrangers, des laboratoires publics ou privés. 


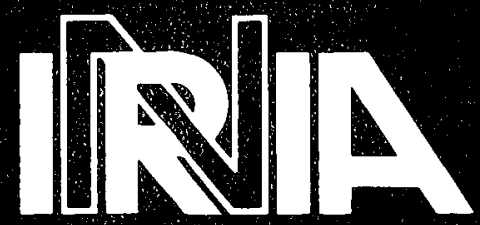

UNITÉ DE RECHERCHE INRIA-ROCOUENCOURT

Instituit National de Recherche en triformatique et en Automatique

Domaine devoluceau

Rocquencourt

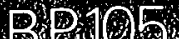

78153 Le 으.

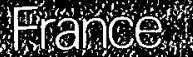

Tél.:(1) 3963551

\section{Rapports de Recherche}

$\mathbf{N}^{\circ} 860$

\section{RECTIFICATION OF IMAGES FOR BINOCULAR AND TRINOCULAR STEREOVISION}

Nicholas AYACHE

Charles HANSEN

JUIN 1988 


\title{
Rectification of Images for Binocular and Trinocular Stereovision
}

\author{
Nicholas Ayache Charles Hansen
}

October 1987

INRIA

BP 105

78153 Le Chesnay Cédex

\begin{abstract}
We present a technique for calibrating and rectifying in a very efficient and simple manner pairs or triplets of images taken for binocular or trinocular stereovision systems. After the rectification of images, epipolar lines are parallel to the axes of the image coordinate frames: therefore, potential matches between two or three images satisfy simpler relations, allowing for simpler and more efficient matching algorithms. Experimental results obtained with a binocular and a trinocular stereovision system are presented and complexity analysis is provided.

Nous présentons une technique pour calibrer et rectifier de manière efficace et simple des couples ou des triplets d'images stéréoscopiques. Après rectification, les droites épipolaires sont parallèles aux axes des repères images: ainsi, les appariements potentiels vérifient des contraintes plus simples, ce qui permet d'accélérer les algorithmes de mise en correspondance. Nous présentons des résultats expérimentaux et précisons la complexité algorithmique de la rectification.
\end{abstract}

Key-words: Rectification, Stereovision, Binocular, Trinocular, Calibration. 


\section{Contents}

1 Introduction $\quad 4$

2 Image Modelling 4

3 Determining the perspective matrix $T$

3.1 Principle ........................ 5

4 General epipolar geometry 6

4.1 Epipolar lines . . . . . . . . . . . . . . . . . . . . 6

4.2 Determining optical centers . . . . . . . . . . . . . . . 7

4.3 Computing inverse images . . . . . . . . . . . . . . . 7

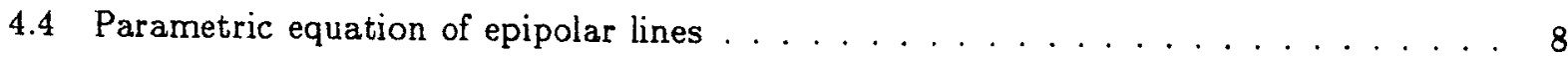

5 Rectification of two images $\quad \cdot 9$

5.1 Getting horizontal epipolar lines . . . . . . . . . . . . . . . 9

5.2 New perspective matrices . . . . . . . . . . . . . . . . 9

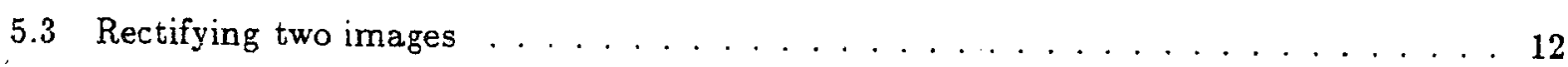

6 Rectification of three images $\quad 13$

6.1 Getting horizontal and vertical epipolar lines . . . . . . . . . . . 13

6.2 New perspective matrices . . . . . . . . . . . . . . . . 13

6.3 Rectifying three images $\ldots \ldots \ldots \ldots \ldots \ldots \ldots \ldots \ldots$

7 Algorithmic complexity $\quad 17$

8 Intrinsic rectification $\quad 17$

9 3D Reconstruction $\quad 19$

$\begin{array}{ll}10 \text { Experimental Results } & 19\end{array}$

$\begin{array}{ll}11 \text { Conclusions } & 20\end{array}$

12 Acknowledgements $\quad 21$

A Estimation of $T \quad \mathbf{2 4}$

A.1 Global least-squares estimation of $T \ldots \ldots \ldots \ldots \ldots \ldots$

A.2 Recursive least-squares estimation of $T \ldots \ldots \ldots \ldots \ldots \ldots$

B Distance of $O^{\prime}$ to $D \quad 25$ 


\title{
Rectification of Images for Binocular and Trinocular Stereovision
}

\author{
Nicholas Ayache Charles Hansen \\ October 1987 \\ INRIA \\ BP 105 \\ 78153 Le Chesnay Cédex
}

\begin{abstract}
We present a technique for calibrating and rectifying in a very efficient and simple manner pairs or triplets of images taken for binocular or trinocular stereovision systems. After the rectification of images, epipolar lines are parallel to the axes of the image coordinate frames: therefore, potential matches between two or three images satisfy simpler relations, allowing for simpler and more efficient matching algorithms. Experimental results obtained with a binocular and a trinocular stereovision system are presented and complexity analysis is provided.

Nous présentons une technique pour calibrer et rectifier de manière efficace et simple des couples ou des triplets d'images stéréoscopiques. Après rectification, les droites épipolaires sont parallèles aux axes des repères images: ainsi, les appariements potentiels vérifient des contraintes plus simples, ce qui permet d'accélérer les algorithmes de mise en correspondance. Nous présentons des résultats expérimentaux et précisons la complexité algorithmique de la rectification.
\end{abstract}

Key-words: Rectification, Stereovision, Binocular, Trinocular, Calibration. 


\section{Contents}

1 Introduction

2 Image Modelling

3 Determining the perspective matrix $T$

3.1 Principle ......................... 5

4 General epipolar geometry 6

4.1 Epipolar lines . . . . . . . . . . . . . . . . 6

4.2 Determining optical centers $\ldots \ldots \ldots \ldots \ldots \ldots \ldots \ldots \ldots$

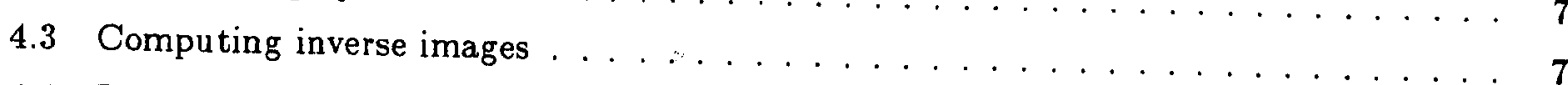

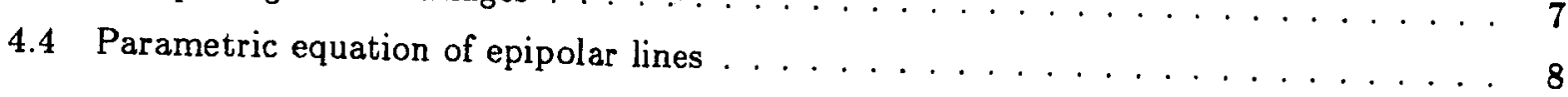

5 Rectification of two images $\quad 9$

5.1 Getting horizontal epipolar lines $\ldots \ldots \ldots \ldots \ldots \ldots . \ldots \ldots$

5.2 New perspective matrices $\ldots \ldots \ldots \ldots \ldots \ldots \ldots \ldots$

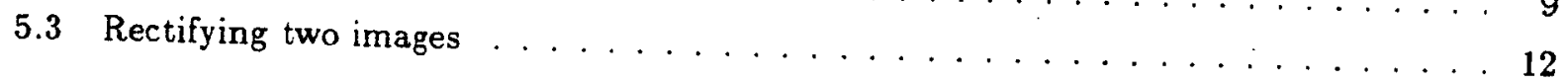

6 Rectification of three images $\quad 13$

6.1 Getting horizontal and vertical epipolar lines ............... 13

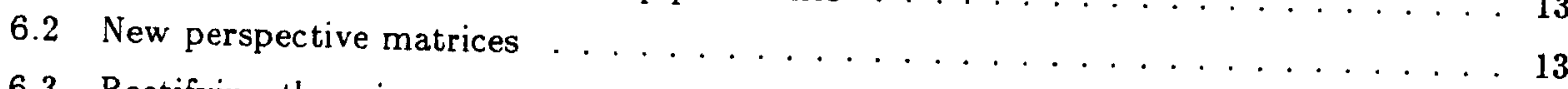

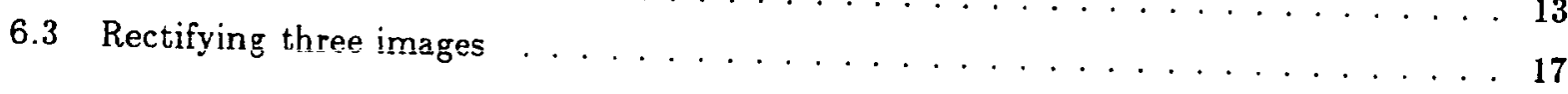

7 Algorithmic complexity $\quad 17$

8 Intrinsic rectification $\quad 17$

9 3D Reconstruction $\quad 19$

10 Experimental Results

11 Conclusions

12 Acknowledgements

A Estinution of $T$

A.1 Global least-squares estimation of $T \ldots \ldots$

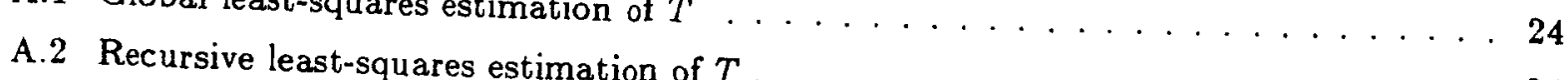

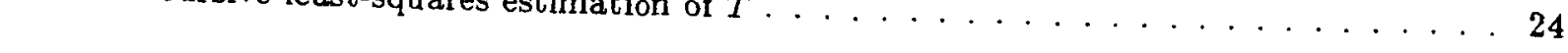

B Distance of $O^{\prime}$ to $D$ 
C 3D reconstruction

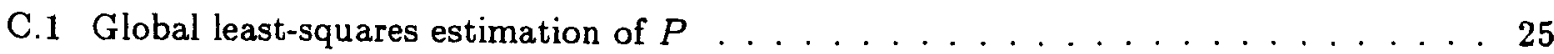

C.2 Recursive least-squares estimation of $P \ldots \ldots \ldots \ldots \ldots$ 


\section{Introduction}

We present in this paper a simple formalism for the calibration of binocular and trinocular stereovision systems which includes the rectification of images for enforcing horizontal and/or vertical epipolar lines which leads to much more efficient stereomatching algorithms.

This rectification could be avoided if images were taken with identical cameras placed in very specific relative positions. In practice, it is impossible, and such assumptions lead to large approximations for the localization of epipolar lines and inaccurate $3 \mathrm{D}$ reconstructions.

Our approach is tied to but different from the ones developed by $[1,2,3,4,5,6,7]$ in the sense that we insist on computing explicitely neither the intrinsic parameters of each camera (focal length, piercing point, horizontal and vertical image units...), neither their extrinsic parameters (rotation and translation with respect to an absolute reference frame), but only the perspective matrices used to relate, in projective coordinates, $3 \mathrm{D}$ scene points to their corresponding $2 \mathrm{D}$ image points.

First, we show how to estimate the perspective matrices through simple least squares procedures. Second we describe the epipolar geometry in the general case. Then we show how to rectify images at a minimal cost to obtain the simplest possible epipolar geometry. This cost is the storage of two $3 \times 3$ matrices in the binocular case or three such matrices in the trinocular case, and then of 6 multiplications, 6 additions and two divisions per rectified point. Finally, we indicate how $3 \mathrm{D}$ reconstruction is performed from the matches obtained on the rectified images. Experimental results obtained with binocular and trinocular stereovision systems are presented.

\section{Image Modelling}

Each camera is modelled by its optical center $C$ and its image plane $P$ (cf. figure 1). This is the classical pinhole model. A point $P$ in the observed scene is projected on point $I$ of the camera retina. Point $I$ is the intersection of the straight line $P C$ with the image plane $P$.

The relationship between $P$ and $I$ is modelled as a linear transformation in projective coordinates. If we denote $I^{*}=(U, V, S)^{t}$ the projective coordinates of $i$ and $(x, y, z)^{t}$ the coordinates of $P$, the following relation holds:

$$
I^{*}=\left(\begin{array}{c}
U \\
V \\
S
\end{array}\right)=T\left(\begin{array}{l}
x \\
y \\
z \\
1
\end{array}\right)
$$

where $T$ is a $3 \times 4$ matrix usually called the perspective matrix of the considered camera.

If $P$ is in the focal plane of the camera, (i.e. if the straight line $C P$ is parallel to the image plane $P$ ), then $S=0$ and the coordinates $(u, v)^{t}$ of $I$ are no longer defined. In the general case 


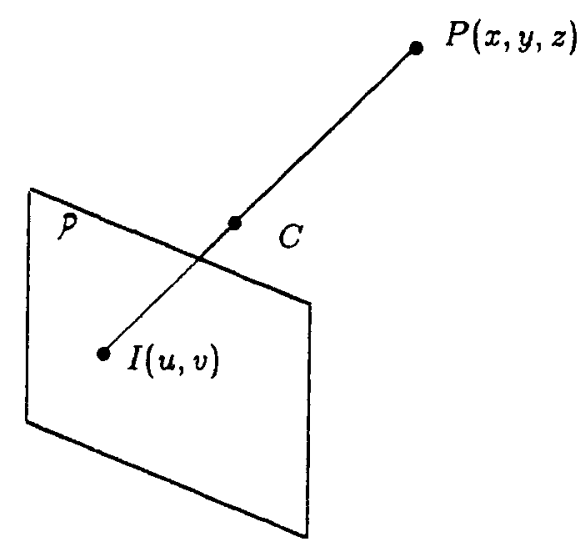

Figure 1: Camera Modelling

$S \neq 0$ and the image coordinates of $I$ (usually expressed in pizels) are given by:

$$
I=\left(\begin{array}{l}
u \\
v
\end{array}\right)=\left(\begin{array}{c}
U / S \\
V / S
\end{array}\right)
$$

\section{Determining the perspective matrix $T$}

\subsection{Principle}

In the experiments conducted in our laboratory $[1,2], T$ is obtained by analysing a calibration pattern which is a grid painted on a planar surface. The 3D position of the intersection points of the grid are well known in an absolute $3 \mathrm{D}$ coordinate frame and the grid is observed from several well defined different positions.

$T$ is a matrix of dimension $3 \times 4$, but it is defined up to a scale factor, and one needs a constraint to specify $T$ uniquely. The simplest constraint ${ }^{1}$ consists in assuming that $t_{34} \neq 0$, then enforcing

$$
t_{34}=1
$$

Each time an image point $I=(u, v)^{t}$ is matched with its corresponding scene point $P=(x, y, z)^{t}$, this provides the following two linear equations on the eleven unknowns remaining for determining $T$ :

$$
\begin{aligned}
& P^{t} t_{1}+t_{14}-u\left(P^{t} t_{3}+1\right)=0 \\
& P^{t} t_{2}+t_{24}-v\left(P^{t} t_{3}+1\right)=0
\end{aligned}
$$

Ion the discussion of this constraint, see $[1,2]$. 
where $t_{j k}$ is the element of rank $(j, k)$ in $T$, and $t_{j}$ is the 3 -vector obtained from the first 3 elements of the $j^{\text {th }}$ row of $T$ :

$$
t_{j}=\left(t_{j 1}, t_{j 2}, t_{j 3}\right)^{t}
$$

In theory, six non coplanar points are sufficient for determining $T$ uniquely [8]. In practice, several dozens of points are available, allowing for a global or recursive least squares estimation of $T$ (see the Appendix).

\section{General epipolar geometry}

We now assume that we are dealing with at least two cameras, called camera 1 and camera 2 , and we compute the relations between them.

\subsection{Epipolar lines}

Given a point $I_{1}$ in image 1 (cf. figure 2), we look for its corresponding point $I_{2}$ in image 2. It appears that $I_{2}$ belongs necessary to a straight line of image 2 entirely defined by the coordinates of $I_{1}$ and the relative geometry of the two cameras, called the epipolar line attached to $I_{1}$.

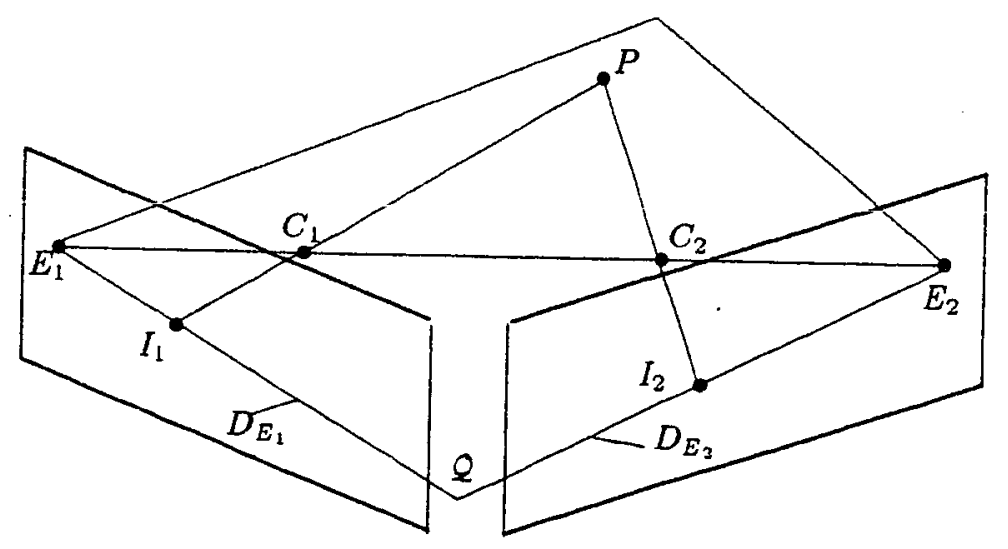

Figure 2: General epipolar geometry

In effect, the set of physical points $P$ whose image corresponds to $I_{1}$ is the straight line $C_{1} I_{1}$ from which we exlude $C_{1}$. The image of this straight line in camera 2 is the epipolar line attached to $I_{1}$. The problem is perfectiy symmetrical, and if one considers the plane $Q$ defined by $I_{1}, C_{1}$ and $C_{2}$, it is clear that this plane intersects the image planes along two straight lines which are conjugated epipolar lines $D_{12}$ and $D_{21}$. Any point $I_{1}$ of $D_{12}$ has its potential corresponding points on $D_{21}$ and vice-versa. 
To compute analytically the equation of the epipolar lines, we need first to compute from each matrix $T_{i}$ the optical center of the cameras and the inverse image of an arbitrary image point.

\subsection{Determining optical centers}

The 3D coordinates $\left(x_{C_{i}}, y_{C_{i}}, z_{C_{i}}\right)$ of the optical center $C_{i}$ of camera $i$ (modelled by the perspective matrix $T_{i}$ ) are obtained by solving:

$$
\left(\begin{array}{c}
0 \\
0 \\
0
\end{array}\right)=T_{i}\left(\begin{array}{c}
x_{C_{i}} \\
y_{C_{i}} \\
z_{C_{i}} \\
1
\end{array}\right)
$$

which is a system of three linear equations in the three unknowns $\left(x_{C_{i}}, y_{C_{i}}, z_{C_{i}}\right)$.

\subsection{Computing inverse images}

We need to compute the straight line $D$ which is the inverse image in the scene of a given image point $I$. This straight line $D$ is composed of $3 \mathrm{D}$ scene points $P$ having the same image $I$. If we look at figure 2 we see that $D$ is simply the straight line defined by $I$ and $C_{i}$.

To determine $D$ analytically, let us re-write the system of equations 1 which relates point $I_{i}$ to points $P$ in the form:

$$
\begin{aligned}
& \left(t_{1}^{i}-u_{1} t_{3}^{i}\right)^{t} P+t_{14}^{i}-u_{1} t_{34}^{i}=0 \\
& \left(t_{2}^{i}-v_{1} t_{3}^{i}\right)^{t} P+t_{24}^{i}-v_{1} t_{34}^{i}=0
\end{aligned}
$$

where the $i$ index in $t_{j}^{i}$ refers to camera $i$.

These equations are nothing else than the equations of two planes whose intersection defines $D$. A vector $n$ colinear to $D$ is the cross-product of the normals to the planes:

$$
n=\left(t_{1}^{i}-u_{1} t_{3}^{i}\right) \times\left(t_{2}^{i}-v_{1} t_{3}^{i}\right)
$$

which yields:

$$
n=u_{1} t_{2}^{i} \times t_{3}^{i}+v_{1} t_{3}^{i} \times t_{1}^{i}+t_{1}^{i} \times t_{2}^{i}
$$

which can be written

$$
n=N_{i} I_{i}^{*}
$$

with

$$
N_{i}=\left[\begin{array}{lll}
t_{2}^{i} \times t_{3}^{i} & t_{3}^{i} \times t_{1}^{i} & t_{1}^{i} \times t_{2}^{i}
\end{array}\right]
$$

The parametric equation of the line $C_{1} I_{1}$ is therefore given by

$$
P=C_{i}+\lambda n
$$

, where $n$ is given by the previous equation and where $\lambda$ is a real number. 


\subsection{Parametric equation of epipolar lines}

It is now easy to compute the parametric equation of the epipolar line $D_{21}$ in image 2 corresponding to the image point $I_{1}$ of coordinates $\left(u_{1}, v_{1}\right)$ in image 1 , because $D_{21}$ is simply the image of the line $C_{1} I_{1}$ by camera 2 . Therefore $D_{21}$ is composed of points $I_{2}$ whose projective coordinates satisfy:

$$
I_{2}^{*}=T_{2}\left(\begin{array}{c}
C_{1}+\lambda n \\
1
\end{array}\right)
$$

If we denote

$$
F_{2}^{*}=T_{2}^{\prime} n
$$

where $T_{2}^{\prime}$ is the $3 \times 3$ sub-matrix obtained from $T_{2}$ by suppressing its last column, and

$$
E_{2}^{*}=T_{2}\left(\begin{array}{c}
C_{1} \\
1
\end{array}\right)
$$

then we get the parametric equation of the epipolar line $D_{21}$ in projective coordinates:

$$
I_{2}^{*}=E_{2}^{*}+\lambda F_{2}^{*}
$$

Therefore, the parametric equation of $D_{21}$ in image coordinates is:

$$
\begin{aligned}
& u_{2}=\frac{U_{E_{2}}+\lambda U_{F_{2}}}{S_{E_{2}}+\lambda S_{F_{3}}} \\
& v_{2}=\frac{V_{E_{2}}+\lambda V_{F_{2}}}{S_{E_{2}}+\lambda S_{F_{2}}}
\end{aligned}
$$

From these equations, it is easy to see that the epipolar lines form a pencil of lines going through an epipolar center $E_{2}$ which is the image of $C_{1}$ in camera 2. One can also notice that a vector colinear to the epipolar line $D_{21}$ is obtained by differentiation of equations 5 and 6 with respect to $\lambda$. This yields

$$
\left(\begin{array}{c}
\Delta u_{2} \\
\Delta v_{2}
\end{array}\right)=\left(\begin{array}{c}
U_{F_{2}} S_{E_{2}}-U_{E_{2}} S_{F_{2}} \\
V_{F_{z}} S_{E_{2}}-V_{E_{2}} S_{F_{2}}
\end{array}\right)
$$

When $S_{E_{2}}=0$, this means that the epipolar center $E_{2}$ is rejected to infinity. In this case, the direction of the epipolar lines becomes independent of the coordinates $\left(u_{1}, v_{1}\right)$ of $I_{1}$, and one can see from equation 7 that in this case all epipolar lines are parallel to the vector:

$$
\left(\begin{array}{c}
\Delta u_{2} \\
\Delta v_{2}
\end{array}\right)=\left(\begin{array}{c}
U_{E_{2}} \\
V_{E_{2}}
\end{array}\right)
$$

We now present a technique which provides new images for which the epipolar lines form such a pencil of parallel lines. 


\section{Rectification of two images}

\subsection{Getting horizontal epipolar lines}

As we have just seen, in the particular case where the image planes $P_{1}$ et $P_{2}$ are coplanar and parallel to the vector $C_{1} C_{2}$ defined by the optical centers, then the epipolar centers are rejected to infinity and the epipolar lines form a pencil of parallel lines. If in addition the image coordinate frames are judiciously defined it is possible that the epipolar line attached to a point $\left(u_{1}^{\prime}, v_{1}^{\prime}\right)$ in image 1 be the line $v_{2}^{\prime}=v_{1}^{\prime}$ in image 2 . We are then in the situation depicted by figure 3 .

We show in this section that it is always possible to apply to each image a transformation which is linear in projective coordinates to obtain conjugated horizontal epipolar lines.

For doing this, let us consider again figure 3 where we have represented the optical centers $C_{i}$ and the image planes $P_{i}$ of each camera. The principle of the rectification is the definition of two new perpective matrices $M$ and $N$ which respectively define the same optical centers $C_{1}$ and $C_{2}$ as $T_{1}$ and $T_{2}$ but with a new common image plane $P^{\prime}$ parallel to $C_{1} C_{2}$. The rectification is the function which computes the new coordinates $\left(u_{i}^{\prime}, v_{i}^{\prime}\right)$ from the old ones $\left(u_{i}, v_{i}\right)$ for each image $i$.

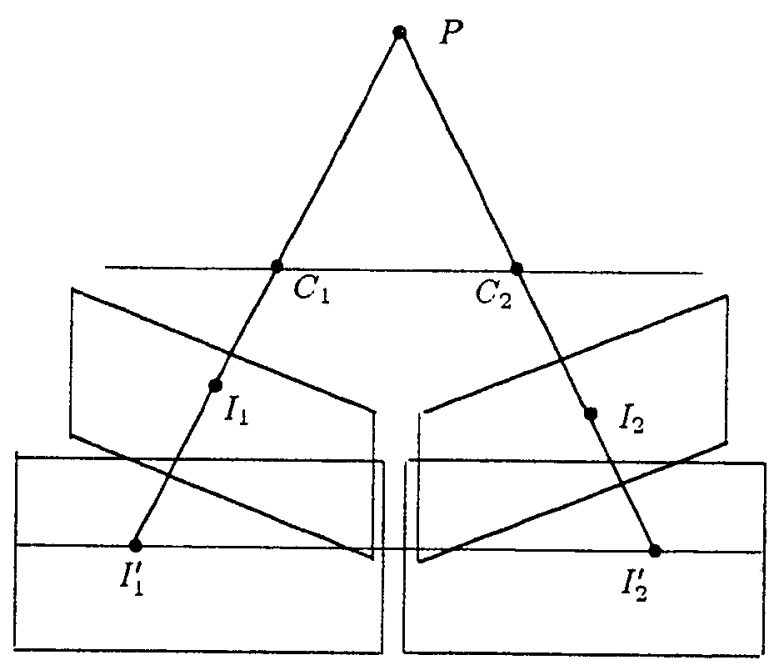

Figure 3: Rectification of two images

\subsection{New perspective matrices}

Let us describe the constraints holding on the new perspective matrices $M$ and $N$.

1. The optical centers of $M$ and $N$ remain respectively $C_{1}$ and $C_{2}$. (this is necessary for having a univoque correspondance between the image points $I_{i}$ and $I_{i}^{\prime}$ respectively before and after rectification). 
2. The focal plane of $M$ must be the same as the focal plane of $N$ (this is necessay for having parallel epipolar lines in the rectified images).

3. For any point $P$ not in this focal plane, the image points $I_{1}^{\prime}$ and $I_{2}^{\prime}$ respectively computed with $M$ and $N$ are such that $v_{1}^{\prime}=v_{2}^{\prime}$ (this is to simplify, as much as possible, the computation of the epipolar lines).

Let us now translate these constraints for the matrices $M$ and $N$. For doing this, we denote.

$$
M=\left(\begin{array}{ll}
m_{1}^{t} & m_{14} \\
m_{2}^{t} & m_{24} \\
m_{3}^{t} & m_{34}
\end{array}\right) ; \quad N=\left(\begin{array}{cc}
n_{1}^{t} & n_{14} \\
n_{2}^{t} & n_{24} \\
n_{3}^{t} & n_{34}
\end{array}\right)
$$

Therefore, one must to estimate 24 parameters. But we have seen that the perspective matrices were defined up to a scale factor. It is therefore possible to choose for instance ${ }^{2}$ :

$$
m_{34}=n_{34}=\left\|C_{1} \times C_{2}\right\|^{2}
$$

There remains 22 parameters to compute to define $M$ and $N$ completely.

Constraint 2 implies that

$$
\forall P \cdot m_{3}^{t} P+\left\|C_{1} \times C_{2}\right\|^{2}=0 \Longleftrightarrow n_{3}^{t} P+\left\|C_{1} \times C_{2}\right\|^{2}=0
$$

Therefore

$$
m_{3}=n_{3}
$$

Moreover, contraint 3 implies:

But, as $m_{3}=n_{3}$, this yields:

$$
\forall P \mid m_{3}^{t} P+\left\|C_{1} \times C_{2}\right\|^{2} \neq 0, \quad \frac{m_{2}^{t} P+m_{24}}{m_{3}^{t} P+\left\|C_{1} \times C_{2}\right\|^{2}}=\frac{n_{2}^{t} P+n_{24}}{n_{3}^{t} P+\left\|C_{1} \times C_{2}\right\|^{2}}
$$

$$
m_{2}=n_{2} \text { and } m_{24}=n_{24}
$$

Finally, constraint 1 can be written as:

$$
M\left(\begin{array}{c}
C_{1} \\
1
\end{array}\right)=N\left(\begin{array}{c}
C_{2} \\
1
\end{array}\right)=\left(\begin{array}{l}
0 \\
0 \\
0
\end{array}\right)
$$

Equations 8 to 11 are summarized by the search of $M$ and $N$ of the form:

$$
M=\left(\begin{array}{cc}
m_{1}^{t} & m_{14} \\
m_{2}^{t} & m_{24} \\
m_{3}^{t} & m_{34}
\end{array}\right) \quad N=\left(\begin{array}{cc}
n_{1}^{t} & n_{14} \\
m_{2}^{t} & m_{24} \\
m_{3}^{t} & m_{34}
\end{array}\right)
$$

\footnotetext{
2assuming that $\left\|C_{1} \times C_{2}\right\| \neq 0$
} 
with the system of constraints

$$
\begin{aligned}
m_{1}^{t} C_{1}+m_{14} & =0 \\
m_{2}^{t} C_{1}+m_{24} & =0 \\
m_{2}^{t} C_{2}+m_{24} & =0 \\
n_{1}^{t} C_{2}+n_{14} & =0 \\
m_{3}^{t} C_{1}+\left\|C_{1} \times C_{2}\right\|^{2} & =0 \\
m_{3}^{t} C_{2}+\left\|C_{1} \times C_{2}\right\|^{2} & =0 \\
m_{34}-\left\|C_{1} \times C_{2}\right\|^{2} & =0
\end{aligned}
$$

In conclusion, equations 12 express 7 linear equations on the 16 remaining parameters defining $M$ and $N$. Thus, there remain 9 degrees of freedom which correspond to some degrees of freedom still available to definitely choose the orientation and the distance of plane $P^{\prime}$ as well as the coordinate frames in the new images.

Let us fix in the simplest manner the remaining degrees of freedom. For instance, let us assume that

$$
m_{14}=m_{24}=n_{14}=0
$$

Therefore, it is clear that the following properties must be satisfied:

1. $m_{1}$ must be orthogonal to $C_{1}$,

2. $n_{1}$ must be orthogonal to $C_{2}$,

3. $m_{2}$ must be orthogonal to $C_{1}$ and $C_{2}$,

4. $m_{3}$ must be orthogonal to $C_{1}-C_{2}$.

To satisfy property 3 , we choose

$$
m_{2}=\left(C_{1} \times C_{2}\right)
$$

Then, to satisfy property 1 , we choose $m_{1}$ orthogonal to $C_{1}$ and also, to avoid a degenerate perspective matrix, $m_{1}$ orthogonal to $m_{2}$, which leads to

$$
m_{1}=\left(C_{1} \times C_{2}\right) \times C_{1}
$$

The same reasoning with property 1 leads to

$$
n_{1}=\left(C_{1} \times C_{2}\right) \times C_{2}
$$

Finally, to satisfy property 4 , we choose $m_{3}$ as the cross product of $C_{1}-C_{2}$ with a vector $u$ such that $m_{3}^{t} C_{1}+\left\|C_{1} \times C_{2}\right\|^{2}=0$ which yields $u=C_{1} \times C_{2}$ and:

$$
m_{3}=\left(C_{1}-C_{2}\right) \times\left(C_{1} \times C_{2}\right)
$$


Therefore, matrices $M$ and $N$ are defined as follows:

$$
\begin{aligned}
& M=\left(\begin{array}{cc}
\left(\left(C_{1} \times C_{2}\right) \times C_{1}\right)^{t} & 0 \\
\left(C_{1} \times C_{2}\right)^{t} & 0 \\
\left(\left(C_{1}-C_{2}\right) \times\left(C_{1} \times C_{2}\right)\right)^{t} & \left\|C_{1} \times C_{2}\right\|^{2}
\end{array}\right) \\
& N=\left(\begin{array}{cc}
\left(\left(C_{1} \times C_{2}\right) \times C_{2}\right)^{t} & 0 \\
\left(C_{1} \times C_{2}\right)^{t} & 0 \\
\left(\left(C_{1}-C_{2}\right) \times\left(C_{1} \times C_{2}\right)\right)^{t} & \left\|C_{1} \times C_{2}\right\|^{2}
\end{array}\right)
\end{aligned}
$$

\subsection{Rectifying two images}

As we saw in section 4.3 an image point $I_{1}\left(u_{1}, v_{1}\right)$ in image 1 comes from a $3 \mathrm{D}$ point $P(x, y, z)$ lying on the 3D straight line $D$ defined by $I_{1} C_{1}$. The parametric equation of $D$ is

$$
P=C_{1}+\lambda n
$$

where $n$, a vector colinear to $D$, is given by equation 2 :

$$
n=u_{1} t_{2}^{1} \wedge t_{3}^{1}+v_{1} t_{3}^{1} \wedge t_{1}^{1}+t_{1}^{1} \wedge t_{2}^{1}
$$

The projective coordinates of the new image $I_{1}^{\prime}$ of $P$ are computed as follows:

$$
I_{1}^{\prime}=\left(\begin{array}{c}
U_{1}^{\prime} \\
V_{1}^{\prime} \\
S_{1}^{\prime}
\end{array}\right)=M\left(\begin{array}{c}
C_{1}+n \\
1
\end{array}\right)
$$

But $C_{1}$ is the optical center of $M$, which means that $M\left(C_{1}, 1\right)^{t}=0$. Therefore, the computation of $\dot{I}_{1}^{\prime}$ is simplified as:

$$
I_{1}^{\prime *}=\left(\begin{array}{c}
U_{1}^{\prime} \\
V_{1}^{\prime} \\
S_{1}^{\prime}
\end{array}\right)=M^{\prime} n
$$

where $M^{\prime}$ is simply the $3 \times 3$ matrix obtained from $M$ by deleting its $4^{\text {th }}$ column. As $n$ is computed by an affine transformation from $\left(u_{1}, v_{1}\right)$, one can compute

$$
R_{1}=\left(\begin{array}{c}
\left(\left(C_{1} \times C_{2}\right) \times C_{1}\right)^{t} \\
\left(C_{1} \times C_{2}\right)^{t} \\
\left(\left(C_{1}-C_{2}\right) \times\left(C_{1} \times C_{2}\right)\right)^{t}
\end{array}\right)\left[\begin{array}{lll}
t_{2}^{1} \times t_{3}^{1} & t_{3}^{1} \times t_{1}^{1} & t_{1}^{1} \times t_{2}^{1}
\end{array}\right]
$$

a $3 \times 3$ matrix to obtain

$$
\left(\begin{array}{c}
U_{1}^{\prime} \\
V_{1}^{\prime} \\
S_{1}^{\prime}
\end{array}\right)=R_{1}\left(\begin{array}{c}
u_{1} \\
v_{1} \\
1
\end{array}\right)
$$


By a perfectly symmetric reasoning, we get:

$$
R_{2}=\left(\begin{array}{c}
\left(\left(C_{1} \times C_{2}\right) \times C_{2}\right)^{t} \\
\left(C_{1} \times C_{2}\right)^{t} \\
\left(\left(C_{1}-C_{2}\right) \times\left(C_{1} \times C_{2}\right)\right)^{t}
\end{array}\right)\left[\begin{array}{lll}
t_{2}^{2} \times t_{3}^{2} & t_{3}^{2} \times t_{1}^{2} & t_{1}^{2} \times t_{2}^{2}
\end{array}\right]
$$

and we have:

$$
\left(\begin{array}{c}
U_{2}^{\prime} \\
V_{2}^{\prime} \\
S_{2}^{\prime}
\end{array}\right)=R_{2}\left(\begin{array}{c}
u_{2} \\
v_{2} \\
1
\end{array}\right)
$$

Therefore, the rectification of images 1 and 2 is reduced to the application of the two linear tranformations in projective coordinates expressed by the equations 15 and 16. After rectification, we have parallel horizontal epipolar lines, as desired, with the nice relationship

$$
v_{1}^{\prime}=v_{2}^{\prime}
$$

\section{Rectification of three images}

\subsection{Getting horizontal and vertical epipolar lines}

For three cameras, it is possible to rectify the images to get horizontal epipolar lines between images 1 and 2, and vertical epipolar lines between images 1 and 3 . For doing this, the image planes $P_{1} P_{2}$ and $P_{3}$ must be coplanar and parallel to the plane defined by the optical centers $C_{1} C_{2} C_{3}$. If, in addition, the image coordinate frames are judiciously defined it is possible that the epipolar line attached to a point $\left(u_{1}^{\prime}, v_{1}^{\prime}\right)$ in image 1 be the line $v_{2}^{\prime}=v_{1}^{\prime}$ in image 2 and the line $u_{3}^{\prime}=u_{1}^{\prime}$ in image 3 . Moreover, it is possible to obtain a very simple relationship between images 2 and 3 of the form $u_{2}^{\prime}=v_{3}^{\prime}$. We are then in the situation depicted by figure 4 . For doing this, one must follow the same reasoning as with two cameras.

\subsection{New perspective matrices}

Let us again precisely specify the constraints on the new perspective matrices $M, N$ and $Q$ :

1. The optical centers of $M, N$ and $Q$ remain respectively $C_{1}, C_{2}$ and $C_{3}$. (this is necessary for having a univoque correspondance between the image points $I_{i}$ and $I_{i}^{\prime}, i=1,2,3$ respectively before and after rectification).

2. The focal plane $₹$ of $M, N$ and $Q$ must be the same (this is necessary for having parallel epipolar lines in the rectified images). 


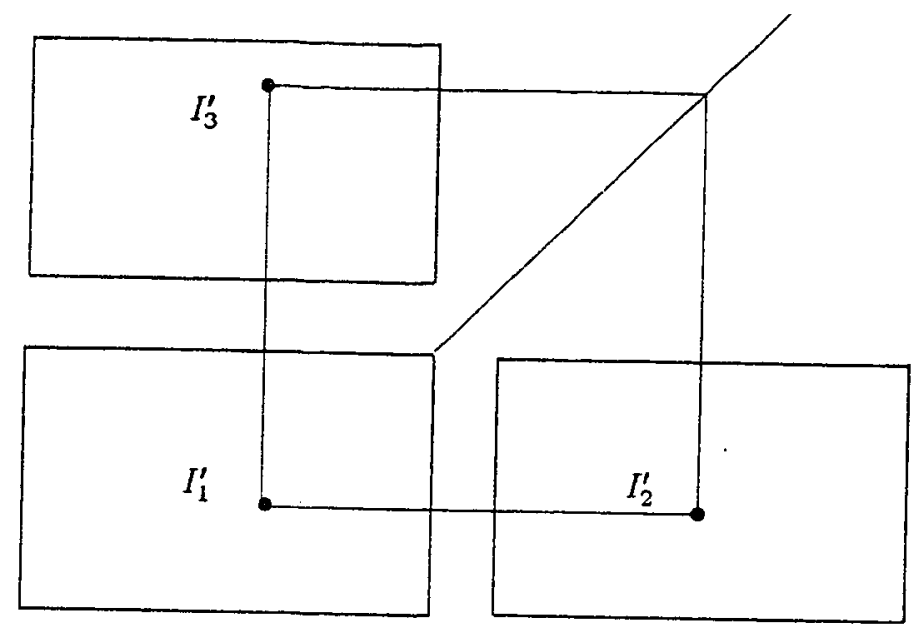

Figure 4: After the rectification of three images

3. For any point $P$ not in the focal plane, the image points $I_{1}^{\prime}, I_{2}^{\prime}$ and $I_{3}^{\prime}$ respectively computed with $M, N$ and $Q$ are such that

- $v_{1}^{\prime}=v_{2}^{\prime}$

- $u_{1}^{\prime}=u_{3}^{\prime}$

- $u_{2}^{\prime}=v_{3}^{\prime}$

Let us now translate these constraints for the matrices $M, N$ and $Q$. For doing this, let us denote $M$ and $N$ as before and:

$$
Q=\left(\begin{array}{ll}
q_{1}^{t} & q_{14} \\
q_{2}^{t} & q_{24} \\
q_{3}^{t} & q_{34}
\end{array}\right)
$$

Therefore one has to estimate 36 parameters. But we have seen that the perspective matrices were defined up to a scale factor. It is therefore possible to choose for instance ${ }^{3}$ :

$$
m_{34}=n_{34}=q_{34}=-\left(C_{1}, C_{2}, C_{3}\right)
$$

where $\left(C_{1}, C_{2}, C_{3}\right)$ is the triple product

$$
\left(C_{1}, C_{2}, C_{3}\right)=\left(C_{1} \times C_{2}\right) \cdot C_{3}
$$

There remains 33 parameters to compute to define $M, N$ and $Q$ completely.

\footnotetext{
${ }^{3}$ assuming that $\left(C_{1}, C_{2}, C_{3}\right) \neq 0$.
} 
Constraint 2 implies that

$$
\forall P \quad m_{3}^{t} P-\left(C_{1}, C_{2}, C_{3}\right)=0 \Longleftrightarrow n_{3}^{t} P-\left(C_{1}, C_{2}, C_{3}\right)=0 \Longleftrightarrow q_{3}^{t} P-\left(C_{1}, C_{2}, C_{3}\right)=0
$$

Therefore

$$
m_{3}=n_{3}=q_{3}
$$

Moreover, constraint 3 implies, as before:

$$
\begin{array}{ll}
m_{2}=n_{2} \text { and } m_{24}=n_{24} \\
m_{1}=q_{1} \text { and } m_{14}=q_{14} \\
n_{1}=q_{2} \text { and } n_{14}=q_{24}
\end{array}
$$

Finally, constraint 1 can be written as:

$$
M\left(\begin{array}{c}
C_{1} \\
1
\end{array}\right)=N\left(\begin{array}{c}
C_{2} \\
1
\end{array}\right)=Q\left(\begin{array}{c}
C_{3} \\
1
\end{array}\right)=\left(\begin{array}{l}
0 \\
0 \\
0
\end{array}\right)
$$

Equations 17 to 22 are summarized by the search of $M, N$ and $Q$ of the form:

$$
M=\left(\begin{array}{cc}
m_{1}^{t} & m_{14} \\
m_{2}^{t} & m_{24} \\
m_{3}^{t} & -\left(C_{1}, C_{2}, C_{3}\right)
\end{array}\right) ; \quad N=\left(\begin{array}{cc}
n_{1}^{t} & n_{14} \\
m_{2}^{t} & m_{24} \\
m_{3}^{t} & -\left(C_{1}, C_{2}, C_{3}\right)
\end{array}\right) ; \quad Q=\left(\begin{array}{cc}
m_{1}^{t} & m_{14} \\
n_{1}^{t} & n_{14} \\
m_{3}^{t} & -\left(C_{1}, C_{2}, C_{3}\right)
\end{array}\right)
$$

satisfying the 10 linear constraints

$$
\begin{aligned}
m_{1}^{t} C_{1}+m_{14} & =0 \\
m_{1}^{t} C_{3}+m_{14} & =0 \\
m_{2}^{t} C_{1}+m_{24} & =0 \\
m_{2}^{t} C_{2}+m_{24} & =0 \\
n_{1}^{t} C_{2}+n_{14} & =0 \\
n_{1}^{t} C_{3}+n_{14} & =0 \\
m_{3}^{t} C_{1}-\left(C_{1}, C_{2}, C_{3}\right) & =0 \\
m_{3}^{t} C_{2}-\left(C_{1}, C_{2}, C_{3}\right) & =0 \\
m_{3}^{t} C_{3}-\left(C_{1}, C_{2}, C_{3}\right) & =0 \\
m_{34}-\left(C_{1}, C_{2}, C_{3}\right) & =0
\end{aligned}
$$

In conclusion, equations 23 express 10 linear equations on the 16 remaining parameters defining $M N$ and $Q$. There remain 6 degrees of freedom which correspond to some degrees of freedom 
still available to definitely choose the distance of plane $P^{\prime}$ as well as some of the parameters of the coordinate frames in the new images.

Let us fix in the most simple way the remaining degrees of freedom. For instance, let us assume that

$$
m_{14}=m_{24}=n_{14}=0
$$

Therefore, it is clear that the following properties must be satisfied:

1. $m_{1}$ must be orthogonal to $C_{1}$ and $C_{3}$,

2. $m_{2}$ must be orthogonal to $C_{1}$ and $C_{2}$,

3. $n_{1}$ must be orthogonal to $C_{2}$ and $C_{3}$,

4. $m_{3}$ must be orthogonal to $C_{1}-C_{2}$ and $C_{1}-C_{3}$.

To satisfy properties 1,2 and 3 , we choose

$$
\begin{aligned}
& m_{1}=\left(C_{3} \times C_{1}\right) \\
& m_{2}=\left(C_{1} \times C_{2}\right) \\
& n_{1}=\left(C_{2} \times C_{3}\right)
\end{aligned}
$$

Then, to satisfy property 4 , we choose

$$
m_{3}=\left(C_{1}-C_{2}\right) \times\left(C_{1}-C_{3}\right)=C_{1} \times C_{2}+C_{2} \times C_{3}+C_{3} \times C_{1}
$$

for which we can easily verify that $m_{3}^{t} C_{1}=m_{3}^{t} C_{2}=m_{3}^{t} C_{3}=\left(C_{1}, C_{2}, C_{3}\right)$.

Therefore, matrices $M, N$ and $Q$ have the following values:

$$
\begin{aligned}
& M=\left(\begin{array}{cc}
\left(C_{3} \times C_{1}\right)^{t} & 0 \\
\left(C_{1} \times C_{2}\right)^{t} & 0 \\
\left(C_{1} \times C_{2}+C_{2} \times C_{3}+C_{3} \times C_{1}\right)^{t} & -\left(C_{1}, C_{2}, C_{3}\right)
\end{array}\right) \\
& N=\left(\begin{array}{cc}
\left(C_{2} \times C_{3}\right)^{t} & 0 \\
\left(C_{1} \times C_{2}\right)^{t} & 0 \\
\left(C_{1} \times C_{2}+C_{2} \times C_{3}+C_{3} \times C_{1}\right)^{t} & -\left(C_{1}, C_{2}, C_{3}\right)
\end{array}\right) \\
& Q=\left(\begin{array}{cc}
\left(C_{3} \times C_{1}\right)^{t} & 0 \\
\left(C_{2} \times C_{3}\right)^{t} & 0 \\
\left(C_{1} \times C_{2}+C_{2} \times C_{3}+C_{3} \times C_{1}\right)^{t} & -\left(C_{1}, C_{2}, C_{3}\right)
\end{array}\right)
\end{aligned}
$$

One can notice that we get the same result as with two cameras for matrices $M$ and $N$ (except for a minor difference in the last line) when identifying $C_{3}=C_{1} \times C_{2}$ in the equations 13 and 14. 


\subsection{Rectifying three images}

It is performed in exactly the same way as before with three $3 \times 3$ rectification matrices called $R_{1}$, $R_{2}$ and $R_{3}$. where

$$
R_{i}=\left(\begin{array}{c}
\left(C_{i-1} \times C_{i}\right)^{t} \\
\left(C_{i} \times C_{i+1}\right)^{t} \\
\left(C_{1} \times C_{2}+C_{2} \times C_{3}+C_{3} \times C_{1}\right)^{t}
\end{array}\right)\left[\begin{array}{lll}
t_{2}^{i} \times t_{3}^{i} & t_{3}^{i} \times t_{1}^{i} & \left.t_{1}^{i} \times t_{2}^{i}\right]
\end{array}\right.
$$

where $i+1=1$ if $i=3$ and $i-1=3$ if $i=1$.

After the rectification of the images we have, as desired, the nice relationships:

$$
\begin{aligned}
& v_{2}^{\prime}=v_{1}^{\prime} \\
& u_{3}^{\prime}=u_{1}^{\prime} \\
& v_{3}^{\prime}=u_{2}^{\prime}
\end{aligned}
$$

which was illustrated by figure 4 .

\section{Algorithmic complexity}

The rectification of $l$ images $(l=2$ or 3$)$ requires the storage of $l 3 \times 3$ matrices, i.e. $9 l$ parameters. Then it requires 6 multiplications, 6 additions and 2 divisions per rectified point.

As the rectification process is a linear transformation in projective space, it preserves straight lines: therefore it is sufficient to apply it to the endoints of the linear segments of a polygonal approximation to get the endpoints of the segments of the rectified polygonal approximation. This is very useful for our stereovision algorithms $[9,10,11]$ which actually deal with linear segments.

\section{Intrinsic rectification}

The matrices $M, N$ and $Q$ used for the rectification of the images depend on the choice of the origin $O$ of the absolute 3D coordinate frame of the scene. The question is how to make the matrices independent of the origin $O$ ?

An answer is the following: it is sufficient to change the origin $O$ of the scene coordinate frame into a point $O^{\prime}$ which is intrinsically defined by the relative geometry of the cameras themselves. For instance, $O^{\prime}$ could be the point which is at a minimum distance from the optical axes of the cameras.

Let us call $D_{i}$ the optical axis of camera $i$, and let us define $D_{i}$ as the set of $3 \mathrm{D}$ scene points whose image is a given point $I_{m}$ chosen in the middle of the image plane. We remember that the parametric equation of $D_{i}$ is given by:

$$
O P=O C_{i}+\lambda n_{i}
$$


where $n_{i}$ is the 3 -vector computed from the coordinates $\left(u_{m}, v_{m}\right)$ of $I_{m}$ by equation 2 :

$$
n_{i}=u_{m} t_{2}^{i} \times t_{3}^{i}+v_{m} t_{3}^{i} \times t_{1}^{i}+t_{1}^{i} \times t_{2}^{i}
$$

The distance of $O^{\prime}$ from $D_{i}$ is given by

$$
d_{i}=\left\|O^{\prime} H_{i}\right\|
$$

where $O^{\prime} H_{i}$ is computed as:

$$
O^{\prime} H_{i}=A_{i} O^{\prime} C_{i}=A_{i} O C_{i}-A_{i} O O^{\prime}
$$

with $A_{i}$ the $3 \times 3$ matrix:

$$
A_{i}=I+\left(n_{i} n_{i}^{t}\right) /\left\|n_{i}\right\|^{2}
$$

and $I$ the $3 \times 3$ identity matrix. The proof is given in the Appendix.

Computing $O^{\prime}$ is then equivalent to minimizing

$$
C=\sum_{i=1}^{k} d_{i}^{2}
$$

(for $k=2$ or 3 ), which is obtained as the least square solution of the linear system of equations:

$$
A O O^{\prime}=b
$$

where

The least square solution is given by

$$
A=\left(\begin{array}{c}
A_{1} \\
\vdots \\
A_{k}
\end{array}\right) \quad \text { and } \quad b=\left(\begin{array}{c}
A_{1} O C_{1} \\
\vdots \\
A_{k} O C_{k}
\end{array}\right)
$$

$$
O O^{\prime}=\left(A^{t} A\right)^{-1} A^{t} b
$$

assuming that $A^{t} A$ has rank 3.

Once $O^{\prime}$ is found, the scene coordinates of any point $P$ are changed into:

$$
O^{\prime} P=O P-O O^{\prime}
$$

and the matrices $T_{i}(i=1, \ldots, k)$ are recomputed for the new coordinate frame, yielding new coordinates for the optical centers

$$
O^{\prime} C_{i}=O C_{i}-O O^{\prime}
$$

and new rectification matrices $R_{i}$, which are now intrinsically defined by the relative geometry of the cameras. 


\section{3D Reconstruction}

Once two points have been matched between original (or rectified) images $\mathrm{i}(i=1,2$ and possibly 3 ), one can use matrices $T_{i}$ 's (resp. $M, N$ and possibly $Q$ ) to compute the $3 \mathrm{D}$ coordinates of the corresponding scene point $P$.

Actually, if we rewrite the system of equations 1 for cameras $i$, one gets a new system of 4 (or possibly 6$)$ equations in the three unknowns $(x, y, z)$ of the $P$ coordinates.

$$
\begin{aligned}
& \left(t_{1}^{i}-u_{i} t_{3}^{i}\right)^{t} P+t_{14}^{i}-u_{i} t_{34}^{i}=0 \\
& \left(t_{2}^{i}-v_{i} t_{3}^{i}\right)^{t} P+t_{24}^{i}-v_{i} t_{34}^{i}=0
\end{aligned}
$$

equations in which the index $i$ of $t_{j}^{i}$ refers to camera $i$ and where $\left(u_{i}, v_{i}\right)$ is replaced by $\left(u_{i}^{\prime}, v_{i}^{\prime}\right)$ if we deal with the rectified images.

In theory these equations are not independent, because the image points are supposed to satisfy the epipolar constraints, and only three independent equations can be extracted from the system. Nevertheless, quantization errors and numerical limited accuracy both vote for a least-squares solution of the entire system. This is detailed in the Appendix.

\section{Experimental Results}

The reprojection technique presented in this paper has been successfully implemented for both binocular and trinocular stereo systems. We have tested this algorithm on a variety of indoor scenes and have found the accuracy of the rectified image to be within 1 pixel for $512 \times 512$ images. Since the rectification takes points (pixels) from the image plane and reprojects them onto a new plane, the rectified points are computed with, arbitrarily fine, sub-pixel accuracy in the rectified space.

Our stereo programs, both 2 and 3 camera $[9,10]$, are feature-based where the features are linear segments. After obtaining a pair or triplet of images, edge points are extracted and chains of connected edges points are built and approximated by linear segments. These segments are the primitives for our stereo matching algorithms and thus are what we rectify. It should be noted that our rectification technique is general in that the rectification is not restricted to segments but can be applied to other types of features or to an entire intensity image thereby improving the matching speeds of different types of stereo programs.

Figure 5 shows a stereo pair of extracted linear segments from a typical room scene. We rectify the segments with the algorithm for two cameras presented in section 5 . The rectified image pair is shown in figure 6 . Note that the epipolar lines form the rows of the images and epipolar conjugates correspond to the same row number. 


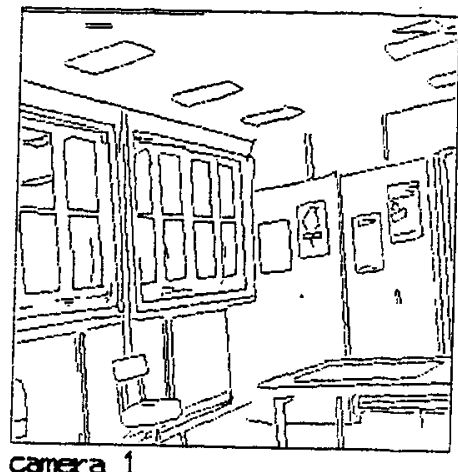

camera 1

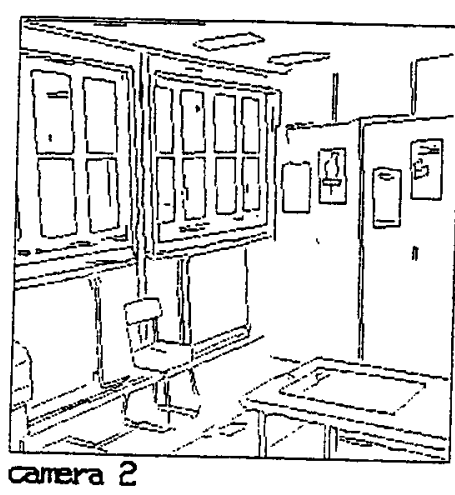

Figure 5: Stereo Pair of Room Scene

We have also tested the rectification algorithm for three cameras. Figure 7 again shows a typical room scene but with a calibrated triplet of cameras. Figure 8 shows the rectified image triplet. Notice that the rectification plane is different in the three camera case than with two cameras. This is because the plane is tilted to include the optical center of the third camera. This results in a slightly more distorted rectified image for some of the cameras, yet the conjugate epipolar lines still maintain the desired relationships: $v_{2}^{\prime}=v_{1}^{\prime}, \quad u_{3}^{\prime}=u_{1}^{\prime}$ and $v_{3}^{\prime}=u_{2}^{\prime}$.

The computational expense for rectification depends on the complexity of the image. On a SUN-3/50 workstation, the computational time for one point is 1.53 millisecond. Thus, for an edge representation of a typical scene, such as figure 5 which contains approximately 400 segments, the computational time is about $0.12 \mathrm{~s}$ on a SUN-3 workstation. We have tested our algorithm on more complex scenes, and as one would expect, we found that the computational time increases linearly with the number of segments. It should be pointed out that this relatively inexpensive operation greatly improves the speed of stereo matching. Since the speed of reliable matching is the highest cost in stereo programs, one can easily justify the expense of rectification. In actual experiments, the time of the stereo matching process was reduced by more than a factor of 2 if the images are rectified before matching $[4,11]$.

\section{Conclusions}

In this paper, we have presented a general and efficient method for the rectification of images used in computational stereo. The method can be used for two or three cameras to enforce simple 


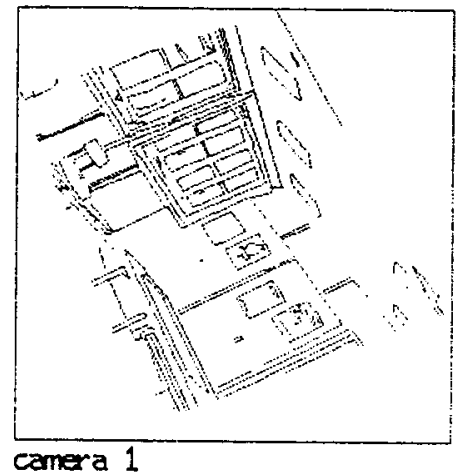

Camera 1

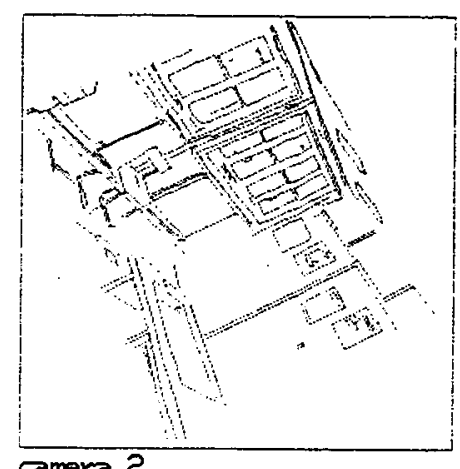

amera 2

Figure 6: Rectified Stereo Pair of Room Scene

constraints on the conjugated epipolar lines in the images.

The presented technique does not rely on difficult, and often inaccurate, mechanical alignment of the cameras. Also, it does not require the explicit computation of the usually called intrinsic and extrinsic parameters of the cameras, but only the simple computation of the perpective matrix of each camera.

After rectification, the epipolar lines are parallel and aligned with the image coordinate frame. Furthermore, conjugate epipolar lines have corresponding row/column numbers. This technique greatly improves the speed of stereo matching by eliminating the need to compute the corresponding epipolar lines in the other image.

Experiments have been presented which have shown the method to be both effective and efficient.

\section{Acknowledgements}

O.D. Faugeras and G. Toscani pioneered the formalism we use for calibration, and the originality of this work comes from the rectification technique. Discussions with both of them and also with Francis Lustman who developed a different rectification technique (using intrinsic parameters of each camera) were very stimulating. 

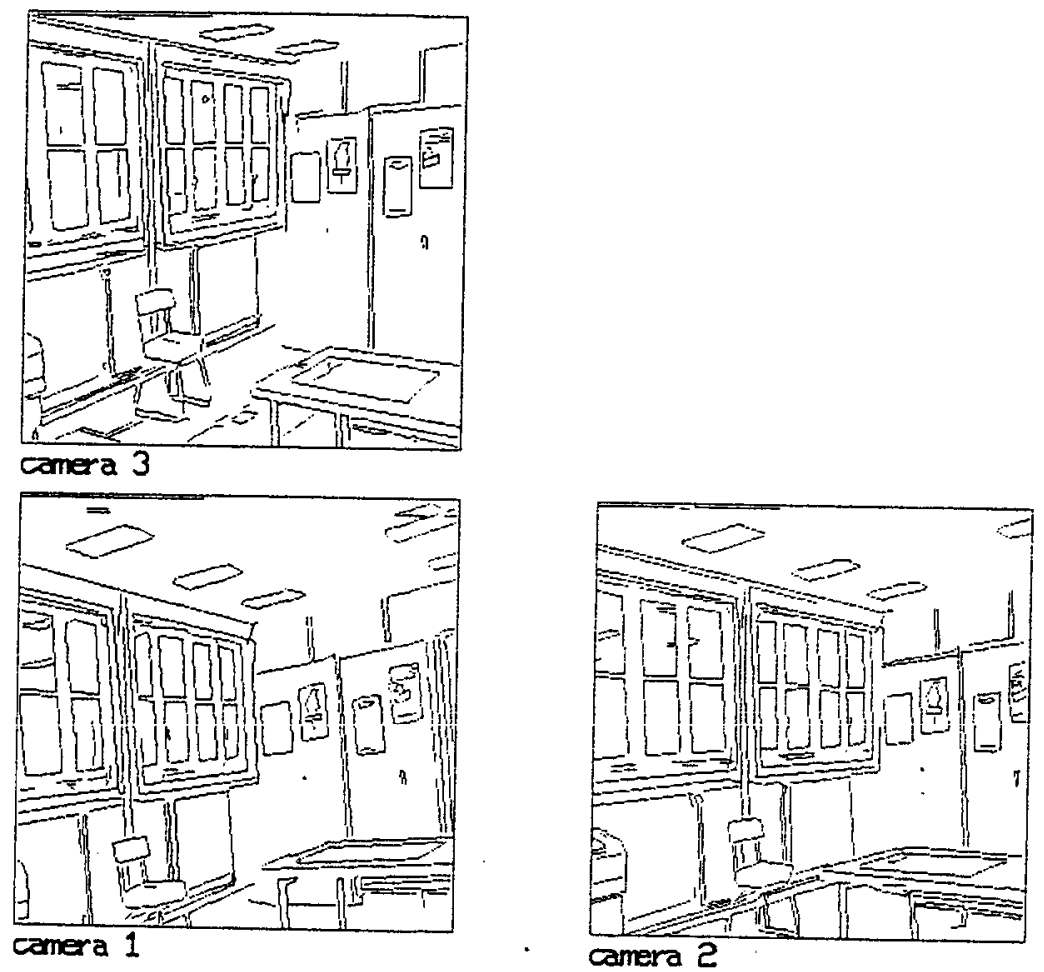

Figure 7: Tripiet of Images of Room Scene 

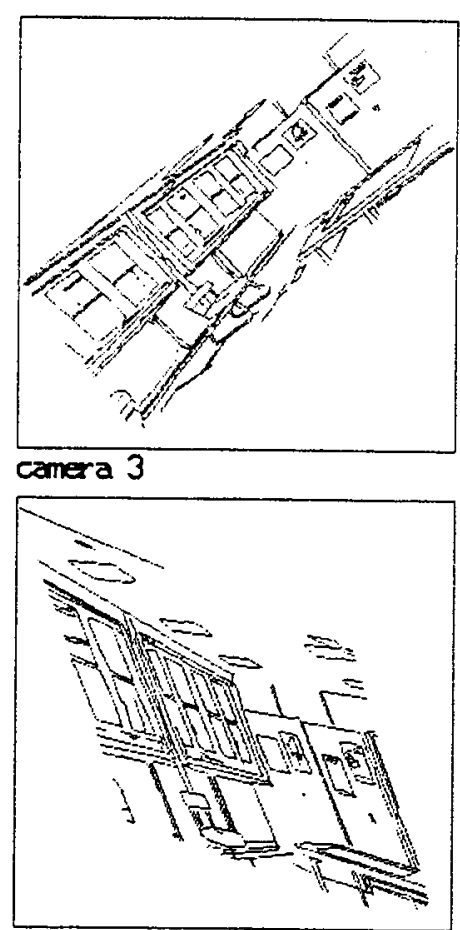

camera 1

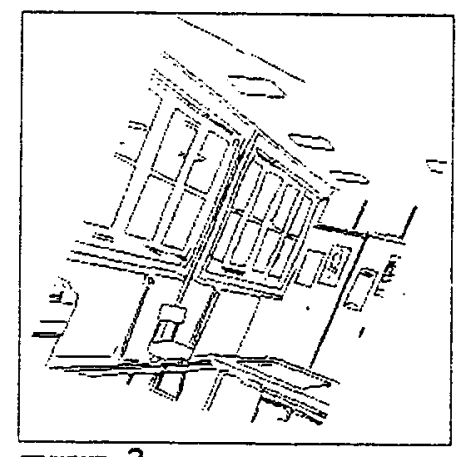

comera ?

Figure 8: Rectified Triplet of Room Scene 


\section{A Estimation of $T$}

\section{A.1 Global least-squares estimation of $T$}

We denote by $a$ the sought parameter vector:

$$
a=\left(\begin{array}{c}
t_{1} \\
t_{14} \\
t_{2} \\
t_{24} \\
t_{3}
\end{array}\right)
$$

If the successively measured $3 \mathrm{D}$ and image points are denoted by $P_{i}$ and $I_{i}(i=1, \ldots, n)$ respectively, this provides the following system of equations:

$$
A a=b
$$

with

for which

$$
A=\left(\begin{array}{c}
A_{1} \\
\vdots \\
A_{n}
\end{array}\right) \quad \text { and } \quad b=\left(\begin{array}{c}
b_{1} \\
\vdots \\
b_{n}
\end{array}\right)
$$

$$
A_{i}=\left(\begin{array}{ccc}
\bar{P}_{i}^{t} & 0_{14} & -u_{i} P_{i}^{t} \\
0_{14} & \bar{P}_{i}^{t} & -v_{i} P_{i}^{t}
\end{array}\right) \text { and } \quad b_{i}=\left(\begin{array}{c}
u_{i} \\
v_{i}
\end{array}\right)
$$

with $\bar{P}_{i}^{t}=\left(x_{i}, y_{i}, z_{i}, 1\right)$ and $0_{14}$ is the null $1 \times 4$ matrix.

The least square solution is given by

$$
a=\left(A^{t} A\right)^{-1} A^{t} b
$$

assuming that $A^{t} A$ has rank 11 .

\section{A.2 Recursive least-squares estimation of $T$}

It is possible to compute a recursive solution of the previous system, and also to take into account the uncertainty on the measurement process. For doing this, one consider $a$ as a state vector and the measurement equations are of the form $A_{i} a=b_{i}+w_{i}$ where $w_{i}$ is related to the noise on the position of the image point $\left(u_{i}, v_{i}\right)$ and on the position of the scene point $P_{i}$. For additional details, one can refer to $[12,13,14]$. 


\section{B Distance of $O^{\prime}$ to $D$}

We compute here the distance $d$ of a point $O^{\prime}$ to the straight line $D$ given by a parametric equation of the form:

$$
O P=O C+\lambda n
$$

Let us call $H$ the orthogonal projection of $O^{\prime}$ on $D$. Clearly,

$$
d=\left\|O^{\prime} H\right\|
$$

As $H$ belongs to $D, O^{\prime} H$ can be written as:

$$
O^{\prime} H=O H-O O^{\prime}=O C+\lambda n-O O^{\prime}=O^{\prime} C+\lambda n
$$

and

$$
\left\|O^{\prime} H\right\|^{2}=\lambda^{2}\|n\|^{2}+2 \lambda n \cdot O^{\prime} C+\left\|O^{\prime} C\right\|^{2}
$$

As $H$ is the point which minimizes the distance to $D$, the value of $\lambda$ is such that

$$
\frac{\partial\left\|O^{\prime} H\right\|^{2}}{\partial \lambda}=0
$$

This yields

and therefore

$$
\lambda=\frac{O^{\prime} C \cdot n}{\|n\|^{2}} n
$$

$$
O^{\prime} H=O^{\prime} C+\left(O^{\prime} C \cdot u\right) u
$$

where

Finally, $O^{\prime} H$ can be written as

$$
u=\frac{1}{\|n\|} n
$$

$$
O^{\prime} H=\left[I+u u^{t}\right] O^{\prime} C
$$

where $I$ is the $3 \times 3$ identity matrix.

\section{3D reconstruction}

\section{C.1 Global least-squares estimation of $P$}

For $n$ cameras, one sets

$$
\dot{A} a=b
$$

with $a=(x, y, z)^{t}$ and

$$
A=\left(\begin{array}{c}
A_{1} \\
\vdots \\
A_{n}
\end{array}\right) \quad \text { and } \quad b=\left(\begin{array}{c}
b_{1} \\
\vdots \\
b_{n}
\end{array}\right)
$$


where ${ }^{4}$

$$
A_{i}=\left(\begin{array}{c}
\left.t_{1}^{i}-u_{i} t_{3}^{i}\right)^{t} \\
\left(t_{2}^{i}-v_{i} t_{3}^{i}\right)^{t}
\end{array}\right) \text { and } \quad b_{i}=\left(\begin{array}{c}
u_{i} t_{34}^{i}-t_{14}^{i} \\
v_{i} t_{34}^{i}-t_{24}^{i}
\end{array}\right)
$$

The least-squares solution is then given by:

$$
a=\left(A^{t} A\right)^{-1} A^{t} b
$$

assuming $A^{t} A$ has rank 3.

\section{C.2 Recursive least-squares estimation of $P$}

Again, it is possible to compute a recursive solution of the previous system, and also to take into account the uncertainty on the measurement For additional details, one can refer to $[13,14]$.

tone must change $\left(u_{i}, v_{i}\right)$ into $\left(u_{i}^{\prime}, v_{i}^{\prime}\right)$ if images are rectified. 


\section{References}

11 G. Toscani. Système de Calibration optique et perception du mouvement en vision artificielle. PhD thesis, Paris-Orsay, 1987.

[2] O.D. Faugeras and G. Toscani. The calibration problem for stereo. In Proceedings CVPR '86, Miami Beach, Florida, pages 15-20, IEEE, 1986.

[3] R. Tsai. An efficient and accurate camera calibration technique for $3 \mathrm{~d}$ machine vision. In Proc. International Conference on Computer Vision and Pattern Recognition, pages 364-374, IEEE, June 1986. Miami Beach, Florida.

[4] F. Lustman. Vision stéréoscopique et perception du mouvement en vision artificielle. $\mathrm{PhD}$ thesis, Paris-Orsay, 1987.

[5] T. M. Strat. Recovering the camera parameters for a transformation matrix. In Proc. Image Understanding Workshop, pages 264-271, October 1984.

[6] B. Caprile and V. Torre. The epipolar transform. 1988. to appear.

[7] G. Gaillat. On the rectification of images. 1988. to appear.

[8] O.D. Faugeras. Artificial SD Vision. To appear, 1988.

[9] N. Ayache and B. Faverjon. Efficient registration of stereo images by matching graph descriptions of edge segments. The. International Journal of Computer Vision, 1(2), April 1987.

[10] N. Ayache and F. Lustman. Fast and reliable passive trinocular stereovision. In Proc. First International Conference on Computer Vision, pages 422-427, IEEE, June 1987. London, U.K.

[11] N. Ayache and F. Lustman. Trinocular stereovision, recent results. In Proc. International Joint Conference on Artificial Intelligence, August 1987. Milano, Italy.

[12] N. Ayache. Construction et fusion de représentations visuelles tridimensionnelles; applications à la robotique mobile. Thèse d'Etat, Université de Paris-Sud, Orsay, May 1988. INRIA Internal Report.

[13] N. Ayache and O.D. Faugeras. Building, registrating and fusing noisy visual maps. In Proc. First International Conference on Computer Vision, pages 73-82, IEEE, June 1987. London, U.K., also an INRIA Internal Report 596, 1986.

[14] N. Ayache and O.D. Faugeras. Maintaining representations of the environment of a mobile robot. In International Symposium on Robotics Research, August 1987. Santa-Cruz, California. 
